\title{
Traffic considerations for security services in VoIP systems
}

\author{
Wilhelm Wimmreuter \\ Siemens $A G$ \\ Information and Communication Networks \\ Hifmannstr. 51, D-81543, Munich, Germany \\ wilhelm.wimmreuter@icn.siemens.de
}

Key words: VoIP, Voice over IP, Security, Traffic, Authentication

Abstract: $\quad$ Voice over IP systems require a new infrastructure of security services. The demand is currently growing and deploys basic-authentication in the first step. Scalable implementations however further evolution of this technologies. This new authentication services using signed certificates and crypto-promise to solve current scalability and management issues. Additionally, if carefully implemented, they can serve for other applications besides VoIP as well. Certificate-authorities, directories and others backend services build the trust environment that delivers the required credentials to network elements, servers and user equipment. All this new backend-services generate a new category of IP traffic.

This paper roughly estimates the expected volume of authentication-traffic and emphasises the importance to select the right services, to handle the possible traffic surge.

\section{INTRODUCTION:}

Traditional public services telephony networks typically provide security based on "Trust by Wire" and therefore have no requirement to utilise other means of security besides the trust imposed by wiring. This is true between network elements and the connection to the customer premises. Of course, tampering with wires is of concern for telephony systems too, but besides 
that and with the exception of special cases for Legal Intercept and Malicious Call Tracing, there are no further provisions necessary.

This situation changes with the transition to the open Internet and Figure 1 gives a brief overview on that.

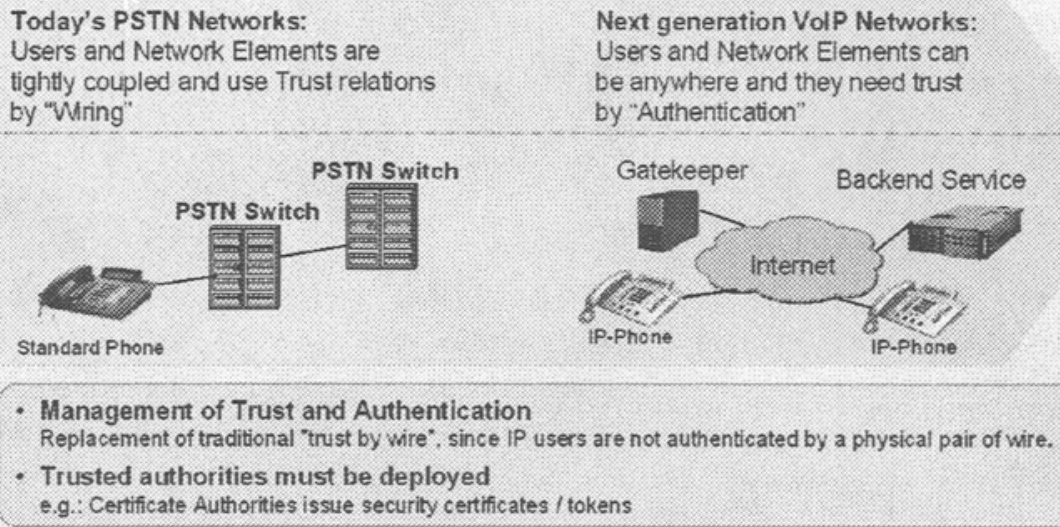

- Management of Trust and Authentication

Replacement of traditional "trust by wire", since IP users are not authenticated by a physical pair of wire.

- Trusted authorities must be deployed

e.g: Certificate Authorities issue security certificates/tokens

Next generation VolP Networks: Users and Network Elements can be anywhere and they need trust by "Authentication"

Figure 1. From Trust-by-Wire to Trust-by-Authentication

Public telephony services for VoIP networks on the other hand can not relay on "Trust by Wire", since the very nature of the Internet does not have the wired trust of traditional phone terminals and network elements. IP networks are build upon "Trust by Authentication". As a major implication, such networks must authenticate their users and their network elements. They must proof each others identity by some means of secure information exchange.

Of course, IP networks for telephony can be operated in secure environments and thus be operated as closed networks. But this will only provide transport technology an is against the goal to provide multi-service networks that can share their resources.

This paper analyses the traffic surge we might expect by introducing new security services for authentication for VoIP users in the internet. Therefore we concentrate to analyse authentication services that need information exchange for every call.

The paper does not consider security related traffic for network element security and other supplementary security services. This simply because of the fact, that these services will not generate bulk-traffic and therefore do not need that kind of attention for traffic engineering. 


\subsection{Elements of VoIP Systems}

To get some common ground we summarise the key elements of VoIP systems.

Figure 2 shows a typical configuration for Carrier VoIP Systems with the following base components:

- Gateways translate media and signalling information between PCM based telephone systems and the internet.

- IP-Phones and Terminal adapters are provide the new customer terminal equipment for VoIP systems.

"Endpoints" is a commonly used term that refers to both, gateways and terminals

- Gatekeepers handle call-control, authentication, authorisation, and billing.

- Backend-Services typically handle the storage of information such as billing, routing, configuration and authentication data for subscribers.

- Clearing-House-Services deal with resources and costs between operators. They are the merchants between multiple operators and fortunately will replace settlement contracts in future.

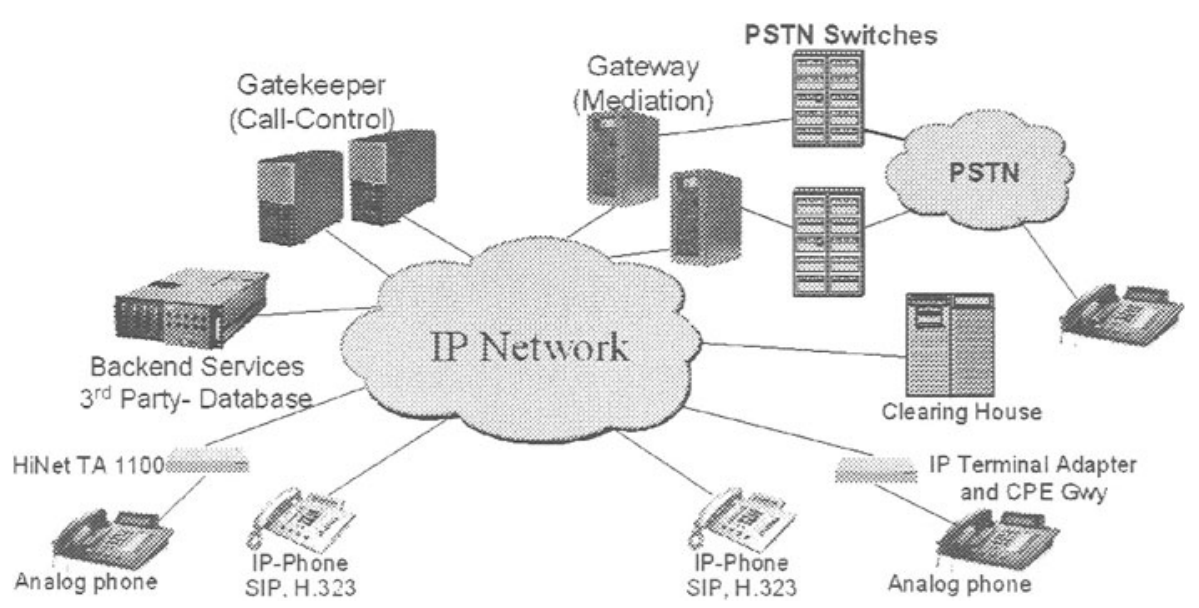

Figure 2. Basic elements of VoIP systems 
IP-phones, Soft-PABX and VoIP-enabled-workstations are the new customer premises terminals for VoIP networks.

IP-terminal-adapters and customer-premises-gateways allow the use of traditional phones, faxes, modems to name a view.

Obviously, these components only provide limited security, and while various standards give guidance on how to implement security on this devices, there is almost nothing that provides a sound environment to support security throughout the network. The missing elements mainly consist of key-management and other backend-services that provide security support for and between above elements.

\subsection{Intelligent Backend - Services for Security}

Backend services for authentication support the basic requirements of security for VoIP systems and must be deployed on the internet. These systems provide all key- and certificate- credentials to authenticate and authorise calls within one and across multiple administrative domains.

Additionally, other security services for media- and signalling- stream encryption, collaborative-work, and transaction-support for E-commerce pave the way for new multi-service-networks. This services go beyond the authentication and authorisation of phone calls. Certainly, invocation of this services is not be done for every call and therefore we must not expect a huge overhead of network traffic generated by this services. 


\subsubsection{Scenarios for VoIP Telephony}

As shown in Figure 2, there are basically five scenarios for telephony on the internet.

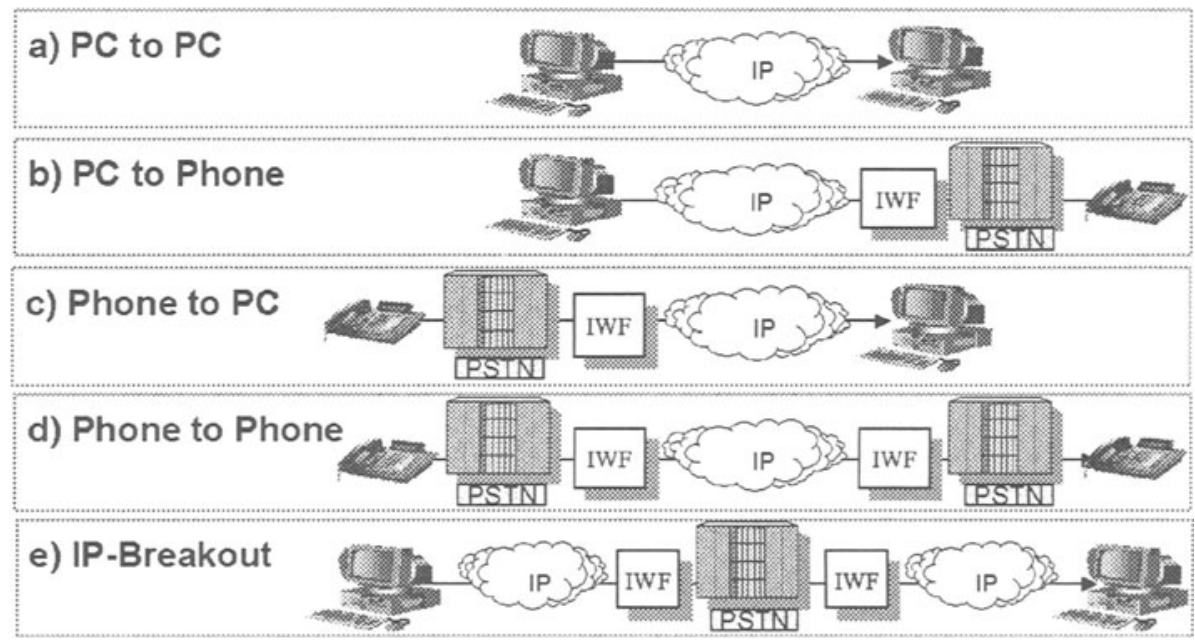

Figure 3. Scenarios for IP-Telephony

a) IP-Endpoint to IP-Endpoint

The Typical PC to PC situation or the situation when VoIP replaces traditional TDM telephony.

b) IP-Endpoint to Traditional Telephony Endpoint

PC or other IP-endpoints calling a standard telephone.

c) Traditional Telephony Endpoint to IP-Endpoint

A telephone subscriber calls a IP-Based endpoint such as PC's or IP-terminals or IP-Phones

d) Traditional Telephony to IP-Traditional Telephony Endpoint The classical Long distance bypass to participate cheaper transmission offered on the internet.

e) IP-Endpoint to IP-Endpoint via PSTN systems (PSTN Breakout) IP endpoints configured to use the PSTN network in case the IP network is overloaded or no peering between IP networks is available.

At present, scenario b) and d) are likely to be the only ones commercially used for public VoIP telephony systems. This is because VoIP still has low penetration and the majority of telephone-subscribers (endpoints) reside in the traditional telephony environment. Of course, there are exceptions where campus-networks replaced their PABX and introduced VoIP in their company. However, this Soft - PABX's typically reside on isolated networks 
where trust and billing is not that much of a concern compared to public telephony.

\subsubsection{Today's authentication for public VoIP telephony}

Out of the major areas of security in VoIP systems, we focus on services for user-authentication. This because identification and authorisation of users is likely to generate an additional traffic surge if not carefully designed.

Other areas of security such as privacy and operational-security deal with encryption and attack prevention. Thus, they deal more with host related issues and may use CPU power, but will not create additional traffic load.

Service providers at present use subscription based authentication and in rare cases credit card based authentication. These services are offered based on prepaid accounts, or authentication and billing through the phone account of the callers local phone provider. Depending on the status of VoIP providers, they either act as licensed interconnect operator in the deregulated telephone market, or based on subscriber like interface in the "not jet" deregulated markets. Fully licensed operators are entitled to use carrierselection-dialling and have the option to bill their users on the phone-bill of the (incumbent) local operator.

The following authentication methods are typically used in today's environments.

Authentication methods for PSTN originated calls:

- Password authentication for a single call

Prepaid card services based in access code dialling with user-ID and password authentication for every call.

- Password authentication for multiple calls

Prepaid card services based in access code dialling with user-ID and password authentication allowing multiple calls once logged in to the Telephony service provider.

- Customer-line-identification based authentication

This method is based on the fact, that gateways and the users access (local telephone switch) maintain a trusted relation. Authentication is based on the service level agreement between the subscriber and the local telephony operator.

Advantage: No extra dialling besides dialling an access-code Disadvantage: No user mobility possible

This authentication methods generate message flows between the PSTNgateway, the call-control-instance, and backend-services of the VoIP operator (proxy, gatekeeper, backend-services). Today, this traffic has no 
effect on the open internet, since gateways and servers are typically interconnected in closed environments or isolated subnets.

As a consequence, no additional authentication messages are required for authentication based on customer-line-identification since the PSTN delivered CLI info is trusted by the VoIP operator.

Authentication methods for Internet originated calls:

- Password authentication for a single call User-ID and password authentication through service selection portals.

- Password authentication for multiple calls User-ID and password authentication through service selection portals.

For this basic password authentication methods, the overhead of message exchange adds up to at least 4 additional roundtrips for MD5 challenge / message-digest exchange. This messages add up to at least $1 \mathrm{k}$ Byte of data transferred for authentication of every single call.

\subsubsection{Authentication services for future demand security}

Scalable systems will need to deploy other authentication services. This because management of trust and authentication must scale for complex interdomain scenarios and trust relations between an ever growing number of operators can not be managed by mutual agreements based on written contracts.

The following technologies promise solutions for the problem:

Security information:

- Public-key authentication.

- Tokens to the endpoint.

- Certificates (X.509)

Security Services:

- Certificate authorities / Trust centres

- Key management systems

- Key revocation systems

- Directories for certificate-authorities 
Such systems can be based on the procedure, that a client authenticates himself with a request signed with the client's private key. The server can then discover the origin of the request if it has access to the public key. The public key shall preferably be signed by a trusted third party. Further, the server my issues a token with limited lifetime to the user. This could avoid re-registration and therefore additional authentication messages for every call.

Figure 4 shows backend security services that support endpoints and servers.
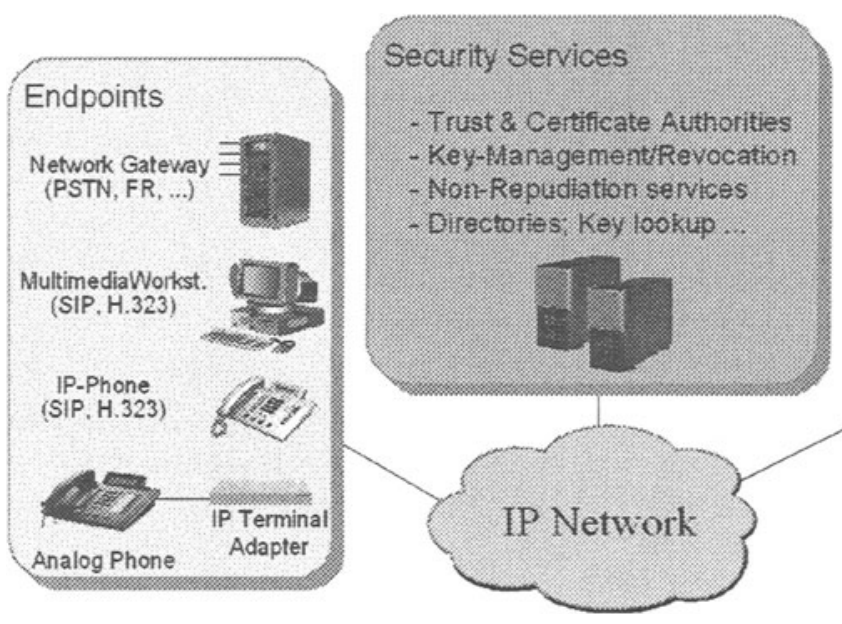

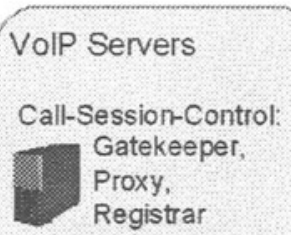

Backend-Services: Billing.

Management, $3^{\text {rd }}$ Party-Db.

Interdomain-Services:

Iocation Service.

Clearing-House...

Figure 4. Authentication Services for IP Telephony

This new backend-services consume bandwidth for their required message exchange. But in case they are carefully designed, a portion of this traffic can be avoided by distributing re-usable information elements with limited lifetime or invocation counters.

Additionally, well thought implementation will open these systems for other applications besides VoIP as well. 


\section{ESTIMATED TRAFFIC GENERATED FOR SECURITY:}

Based on the framework given in section 1, the following estimates are based on the average traffic produced in a city with about 250.000 traditional telephony subscribers. We compare basic signalling and the overhead of traffic expected by introducing IP-based authentication as mentioned above. It is assumed further, that authentication source of security traffic in VoIP networks. Signalling- and security- traffic adds to the top of basic traffic generated by media stream transmission.

\subsection{Media stream traffic volume}

The given call distribution as in Figure 5 shows, that around 1/3'rd of the calls is to be served in the three hours around the busy-hour of the day. Thus, the major bandwidth concerns may arise at that time. This are the most important values to evaluate for network dimensioning. The Busy-HourCall-Attempts (BHCA) and the Mean-Hold-Time (MHT) are the key values in dimensioning this networks. All estimates is based on 200.000 BHCA as shown in Figure 5 and all following bandwidth considerations are based on a given Mean-Hold-Time of $120 \mathrm{Sec}$.

\section{Call Distribution}

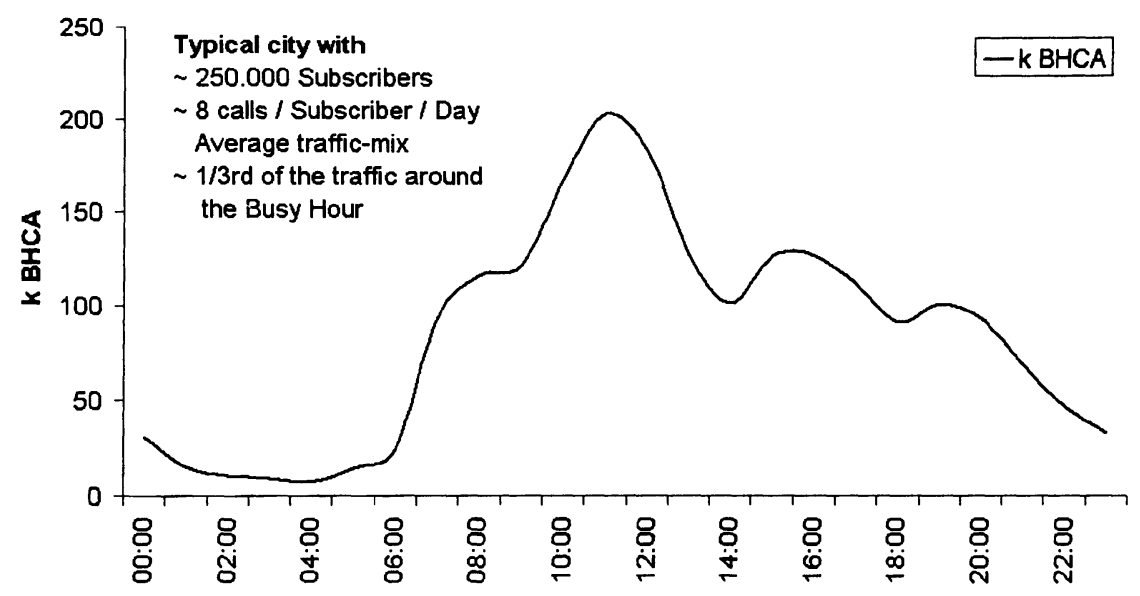

Figure 5. Traffic volume for 250.000 subscribers 
Media-streaming traffic volume as shown in Figure 6 is derived based on traditional telephony methods. Thus poisson distributed queuing for telephony according to "Erlang" is assumed to fit for IP-based voice streams as well.

The bandwidth consumption is based on the measurement in Figure 5 with a set of bandwidths derived from the measured PSTN 64-Kbit/Sec stream.

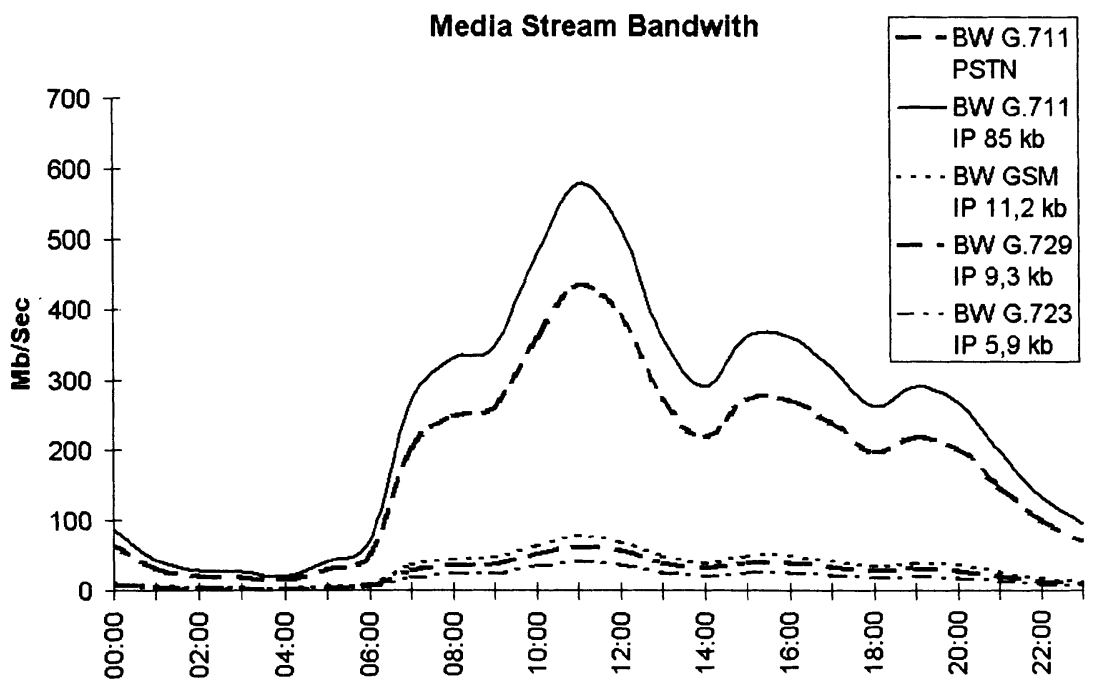

Figure 6. Media stream bandwidth requirement

Figure 6 shows the peak bandwidth requirement of the given PSTN network at about $450 \mathrm{Mbits} / \mathrm{Sec}{ }^{1}$ In the busy-hour. VoIP telephony without compression adds to almost $600 \mathrm{Mbits} / \mathrm{Sec}$. and compressed IP transmission goes down to about one tenth of the PSTN bandwidth trading in some quality to reach this goal.

At present, there is a tendency against compression, since bandwidth is available at large and impacts of compression to FAX and other modem traffic turns out to be a problem in telephony environments. Additionally, IPR and license fees are quite unpredictable for suppliers as well as for operators.

${ }^{1}$ Peak bandwidth in the given PSTN network does not imply the necessity to provide all the bandwidth. In fact, these systems are typically designed with about $30 \%$ of loss on a given peering. This can be done, since e.g. residential-areas might not have the same busy-hour as business-areas. Overflow through temporarily unloaded peerings can save in this case 


\subsection{Signalling traffic volume}

Signalling traffic adds up to the VoIP media traffic budget. Figure 7 shows the comparison of best- and worst- case IP based signalling scenarios with SS7 signalling ${ }^{2}$.

\section{Signaling Traffic Volume}

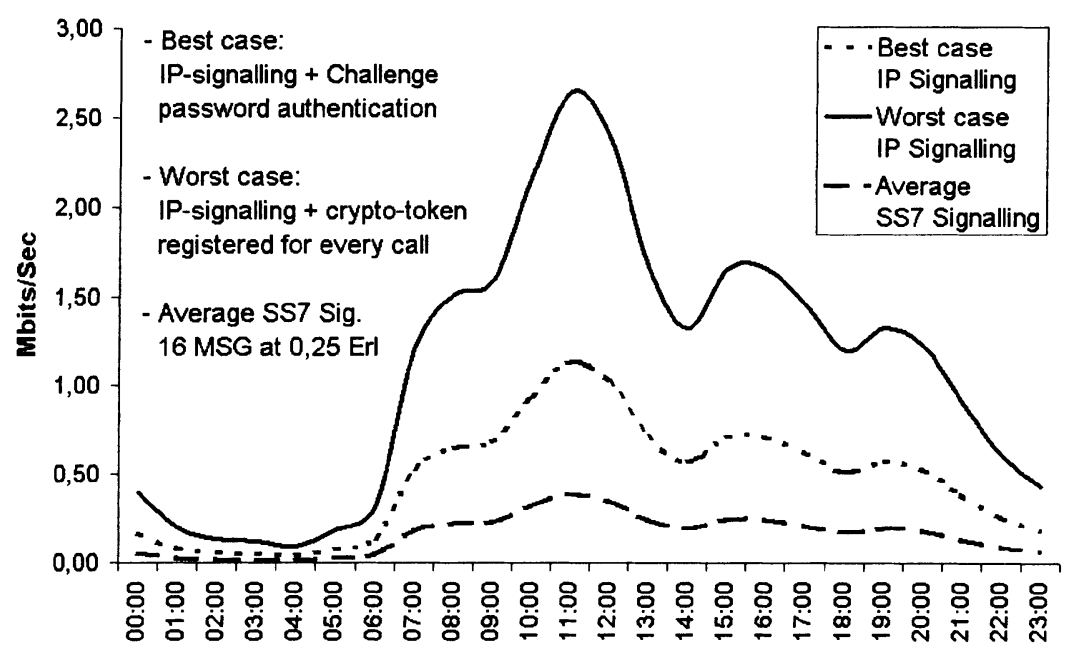

Figure 7. Basic-call signalling bandwidth requirement

Assumptions to simulate signalling for VoIP

- The difference between H.323 and SIP for basic-call signalling is small and therefore ignored (1020 vs. 1040 bytes).

- Only two cases of authentication are considered Basic authentication with challenged passwords for every call, and Crypto-token authentication with re-registration for every call

- 6 messages are assumed for IP-Basic-Call-setup and release

- 4 or 11 messages for IP-Authentication depending on services used.

The graph in Figure 7 shows, that SS7 is the most economic signalling method. However, compared to the media streaming requirements, signalling traffic adds up to at most $0,5 \%$ of media streaming.

${ }^{2}$ Note: Since SS7 is considered pure interoffice trunk signalling and VoIP typically serves for both, trunk and endpoint traffic, a rather complex call was taken in to consideration for the SS7 part of signalling (Basic-call: $8 \mathrm{Msg}$ with 27 Bytes; Used: $16 \mathrm{Msg}$ with 90 Bytes). This shall cover quite complex subscriber signalling cases. 


\subsection{Authentication overhead}

Looking at the worst case of authentication traffic, about $2 \mathrm{Mbits} / \mathrm{Sec}$ of additional traffic is needed for authentication with crypto-tokens that register for every call. Further it is assumed, that each token must be signed by the certificate-authority acting as an independent 3'rd party trust-centre. Of course, this is worst case and according to above traffic estimates, this might be unwise to implement. On the other hand, as long as volume of VoIP traffic is low, a limited lifetime of crypto-tokens is likely to run out anyhow.

Basic clear-password-authentication, and locally resolved challengeauthentication are not considered in this estimate. This because of low security for the clear-password method, and severe scaling problems for the locally resolved challenge method. Therefore 11 message-roundtrips are assumed for to invoke certificate-authorities. This seems to be the only way to design re-usable security services for other applications besides VoIP telephony.

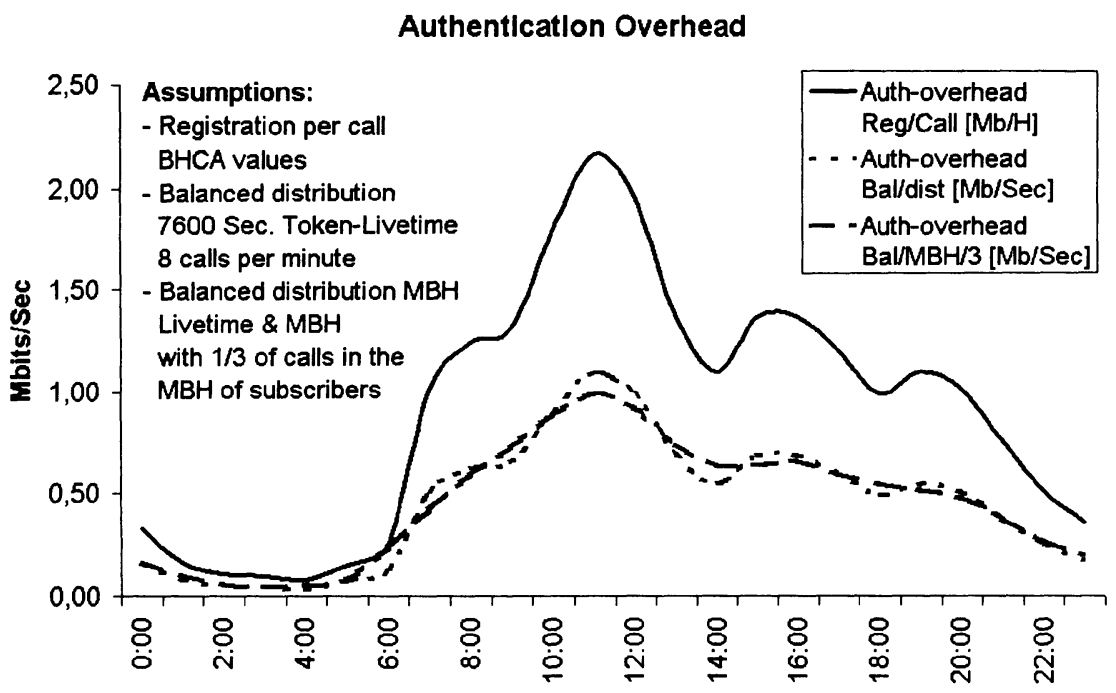

Figure 8. Optimising the authentication overhead

The scenarios according to Figure 8 are based on 2 hour lifetime for the crypto-token, an average of 8 phone calls per subscriber per day, and the fact, that $1 / 3^{\text {rd }}$ of the calls are done in the 3 hours around the busy hour of every subscriber. 
Compared to the total volume of signalling-Traffic in Figure 7, the graph in Figure 8 shows that the majority of signallin $r$ traffic for the basic-call is produced by authentication.

Graphs in Figure 8 provide the following results:

- Registration for each call with access to the certificate-authority: This provides for the worst case with more than $2 \mathrm{Mbits} / \mathrm{second}$ in the busy-hour.

Of course this is likely the most secure method as well.

- Balanced distribution of re-registration at the end of lifetime: Generates less than $50 \%$ of the traffic compared to the previous one.

- Balanced distribution of re-registration considering busy-hour: Here another optimisation considered the fact, that $1 / 3^{\text {rd }}$ of the calls are done around the mean-busy-hour of the subscriber. Therefore a running-average forces (re-) registration at the begin of the mean-busy hour and therefore saves one registration around the busy-hour. This test did not result in real savings, since it only flattens the peaks a little bit. Further tuning to better guess the start of busy hour and the modification of the lifetime of tokens did not further improve results. In consequence, considering all the costs for implementation and management achieving this small the small gain seems to be not worth the effort.

\section{CONCLUSION:}

As shown, the transition to public VoIP networks has major impact to the network traffic. As a consequence, network design, service deployment and business cases must be adopted to this new environment.

Security services make up an essential piece of this puzzle. In fact, this services and authentication in particular produce about 10 times as much signalling traffic compared to signalling traffic in traditional telephony systems.

Trading in media stream bandwidth for signalling seems to be a winning solution. But we shall be careful since this might end in arbitrage business that lasts for a short while only. More advanced solutions will take the benefits of new business cases possible through advanced authentication as required for VoIP. 
Since authentication services must be implemented for VoIP, scalability and re-use of authentication services for other applications such as Ecommerce, banking and others is feasible.

Certificate-authorities and registration-authorities possibly managed by independent 3 'rd party operators, public directories, and encryption are some of the basic services that open the way for multi-purpose authentication. Only this value add my justifies the extra cost of advanced authentication services.

Management of trust and authentication must become scalable and secure. This includes directory-lookup, key-generation, key-management and services providing key-revocation to resolve quite problems around compromised security information.

Finally, if we implement all this security-systems for VoIP without considering the re-use for additional applications, we will have to ask ourselves whether security is affordable for a pure phone call.

\section{REFERENCES}

IETF Working Groups http://www.ietf.org/html.charters/

ETSI TIPHON documents (open access) http://www.etsi.org/tiphon/

GMD Focus Collection of References \& Papers

http://www.fokus.gmd.de/research/cc/glone/projects/ipt/

H.323 Protocol suite: Current documents of SG16

ftp://standard.pictel.com/avc-site/

ITU-T Recommendation H.235 Version 1, Security and Encryption for H Series

(H.323 and other H.245 based) multimedia terminals, 1998.

SIP Web Page at Columbia University

http://www.cs.columbia.edu/sip/

Henning Schulzrinne et. al.

Telephone Traffic Theory, Tables \& Charts

Siemens AG

ISBN 3-8009-1343-7 